\title{
An amphiphilic-DNA platform for the design of crystalline frameworks with programmable structure and functionality.
}

\author{
Ryan A. Brady, ${ }^{\dagger}$ Nicholas J. Brooks, ${ }^{\ddagger}$ Vito Foderà, ${ }^{\uparrow}$ Pietro Cicuta, ${ }^{*}{ }^{\dagger}$ and Lorenzo \\ Di Michele ${ }^{*} \dagger$ \\ $\dagger$ Biological and Soft Systems, Cavendish Laboratory, University of Cambridge, Cambridge \\ CB3 OHE, UK \\ $\ddagger$ Department of Chemistry, Imperial College London, London SW7 2AZ, UK \\ \Department of Pharmacy, University of Copenhagen, Universitetsparken 22100 \\ Copenhagen, Denmark \\ E-mail: pc245@cam.ac.uk; Id389@cam.ac.uk
}

\section{Abstract}

The reliable preparation of functional, ordered, nanostructured frameworks would be a game changer for many emerging technologies, from energy storage to nanomedicine. Underpinned by the excellent molecular recognition of nucleic acids, along with their facile synthesis and breadth of available functionalizations, DNA Nanotechnology is widely acknowledged as a prime route for the rational design of nanostructured materials. Yet, the preparation of crystalline DNA frameworks with programmable structure and functionality remains a challenge. Here we demonstrate the potential of simple amphiphilic DNA motifs, dubbed $C$-stars, as a versatile platform for the design of programmable DNA crystals. In contrast to allDNA materials, in which structure depends on the precise molecular details of individual building blocks, the self-assembly of C-stars is controlled uniquely by their topology and symmetry. Exploiting this robust self-assembly principle we design a range of topologically identical, but structurally and chemically distinct C-stars that following a one-pot reaction selfassemble into highly porous, functional, crystalline frameworks. Simple design variations allow us to fine-tune the lattice parameter and thus control the partitioning of macromolecules within the frameworks, embed responsive motifs that can induce isothermal disassembly, and include chemical moieties to capture target proteins specifically and reversibly.

\section{Introduction}

The production of functional crystalline frameworks is arguably the ultimate goal of DNA nanotechnology, and surely one of its most applicable outcomes. ${ }^{1,2}$ Methodologies have been developed to create a variety of DNA-based arrays with high spatial resolution in $1 \mathrm{D}^{3-6}$ and $2 \mathrm{D},{ }^{6-16}$ and a number of routes exists to extend geometrical control to the third dimension. ${ }^{6,15-20}$ None of the available approaches, however, has been able provide a general route for the preparation of 3D DNA frameworks that combine high porosity, embedded functionality, robustness to design changes, and the ability to retain local order over large lengthscales. Frameworks with these characteristics would enable the production of programmable materials in which functional macromolecules, quantum dots, fluorophores, or non-structural DNA motifs are precisely arranged in space, 
revolutionizing areas of advanced technology including energy storage, ${ }^{21,22}$ photonics, ${ }^{23}$ sensing, ${ }^{24-26}$ and molecular sieving. ${ }^{27}$

Building units in the form of tensegrity triangles have been shown to form diffracting single crystals with a remarkable $\sim 4 \AA$ resolution, and overall dimensions exceeding $100 \mu \mathrm{m} .{ }^{20}$ However, the compact geometry and rigidity of these building blocks, and the required bond directionality, places substantial limits on the accessible rage of free volume, lattice parameters, and the possibility of embedding bulky responsive motifs. ${ }^{20,28}$ Approaches based on DNA “origami' and single-stranded (ss) DNA "bricks", can produce 3D architectures of arbitrary shape, which are however very compact, limiting diffusion of solutes and leaving little space to incorporate active elements or molecular cargo. ${ }^{17-19,29,30}$ An exception is represented by open origami frameworks ${ }^{18}$ and by the recently introduced origami-based tenesegrity triangles that form a scaled-up version of the analogous nanoscale lattices, ${ }^{20,28}$ enabling the incorporation of nanoparticles. ${ }^{31}$ In all cases, the complexity of origami requires cumbersome sample preparation protocols leading to low yields and high costs. Furthermore, as for wireframe DNA lattices, ${ }^{29}$ these approaches have not yet been shown to produce periodic arrays over macroscopic lengthscales.

Here we demonstrate how a platform based on newly introduced amphiphilic DNA nanostructures, dubbed $C$-stars, ${ }^{32}$ can overcome the aforementioned limitations, offering a one-pot route for the formation of highly porous, bulk crystalline mesophases able to robustly support changes in structure and functionality. Rather than depending on the fine geometrical details of the building blocks, the crystal structure of C-star frameworks is prescribed solely by the topology and symmetry of the amphiphilic DNA motifs. ${ }^{32}$ We exploit this unique feature to produce a range of topology-preserving $C$ star designs forming a variety of frameworks with distinct structure and functionalities that nevertheless share the same crystal geometry. We can precisely and continuously tune lattice parameter and framework mesh size, which allows us to control the partitioning of a range of macromolecules. Building blocks tagged with a ligand form frameworks that can specifically and reversibly entrap a target protein, while the use of C-stars modified with a responsive DNA motif leads to triggered disassembly of the frameworks. Furthermore, the resilience of the self-assembly mechanism of C-stars makes crystallization robust to low-ionic strength conditions and to the use of unpurified components.
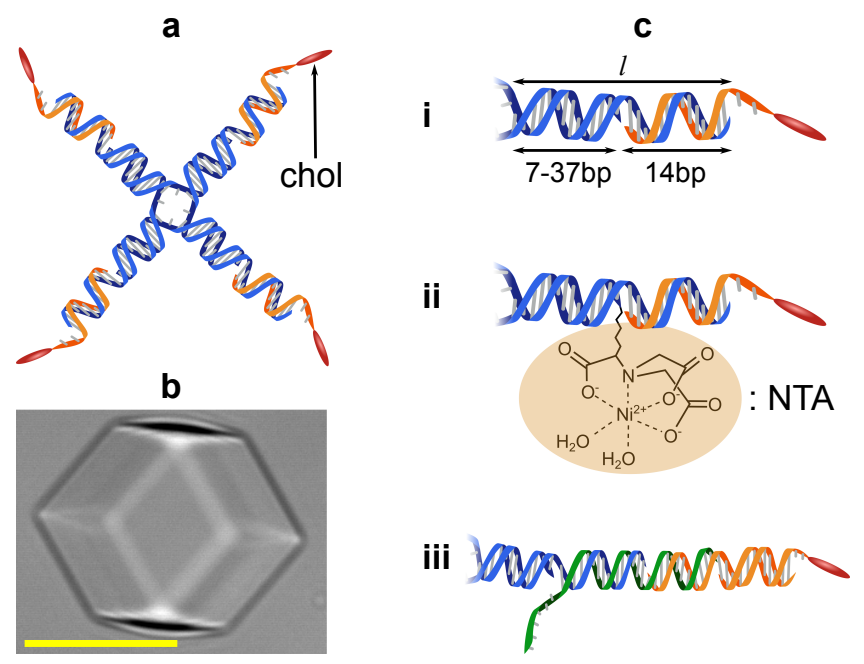

Figure 1: Topology preserving modifications in amphiphilic C-stars. a, C-stars are composed of 4 core-forming oligonucleotides (blue) and 4 cholesterol-functionalized strands (orange). b, Slow cooling of C-star samples leads to the formation of single crystals. Scale bar $30 \mu \mathrm{m}$. c, Topology-preserving modifications include; (i) controlling arm length, (ii) inclusion of functional groups, here nitrilotriacetic acid (NTA), (iii) inclusion of DNA strand-displacement motifs.

\section{Results and Discussion}

\section{Topology-preserving modifications of C-star motifs}

C-stars are simple amphiphilic motifs based on DNA nanostars, ${ }^{33-35}$ composed of 4 coreforming oligonucleotides and 4 cholesterolfunctionalized strands (Fig. 1a). ${ }^{32}$ Slow cooling of a stoichiometric mixture of these components leads to the self-assembly of macroscopic single crystals in a straightforward one-pot re- 
action (Fig. 1b). ${ }^{32}$ In contrast to approaches reliant on Watson-Crick and/or stacking interactions alone, ${ }^{20,28,29,31}$ where a successful selfassembly requires the fine tuning of the building block geometry with atomic resolution, in Cstar frameworks long-range order emerges from the frustrated phase separation between the hydrophobic cholesterol and the hydrophilic DNA. This mechanism is controlled uniquely by the topology and symmetry of the flexible nanostar motif, ${ }^{32}$ which makes C-star self-assembly potentially robust against substantial design changes that preserve these features.

To challenge this hypothesis we designed a range of structurally and chemically distinct C-star sub-units which share the same 4-arm topology. ${ }^{32}$ The investigated design variations are presented schematically in Fig. 1c, and include: (i) a range of C-stars with different arm length designed to program the lattice parameter of the frameworks; (ii) a C-star modified with a chemical group to specifically capture and reversibly release target proteins; (iii) a C-star modified with a responsive DNA strand-displacement motif which can be triggered to induce isothermal dissolution of the frameworks.

\section{Tuning lattice parameter}

While retaining the same 4-arm topology shown in Fig. 1a, we vary nanostar arm length $l$ between 21 and 51 base-pairs (bp). $l$ is controlled by changing the length of the core-forming strands while keeping that of the cholesterolized strand fixed, as shown in Fig. 1c(i). Correct folding of individual nanostar motifs was confirmed by Agarose Gel Electrophoresis (AGE) and Dynamic Light Scattering (DLS), using soluble building blocks in which the cholesterol-modified strands were replaced by non-functionalized ones of identical sequence. AGE produces single sharp bands for arm lengths of 24-51 bp, while nanostructures with $l=21$ give rise to a more smeared band, indicating that such small junctions are not individually stable in running buffer (see Fig. S1 in SI). Fig. 2a demonstrates how the relative electrophoretic migration distance $R_{\mathrm{f}}$ decreases linearly with $l$ in the range $24-51 \mathrm{bp}$, deviating for $l=21 \mathrm{bp}$. A consistent trend is observed in the mean hydrodynamic diameter $\left\langle D_{\mathrm{h}}\right\rangle$ measured by DLS, increasing with $l$.

For C-star samples with $l=21-42$ bp we observe the formation of equilibrium single crystals that, irrespective of arm length, feature a rhombic dodecahedral geometry consistent with an underlying cubic symmetry (Fig. 2b, left). ${ }^{36}$ The relationship between the C-star arm length and the microscopic structure of the self-assembled aggregates was investigated by Small Angle X-ray Scattering (SAXS). Diffraction profiles obtained from "powder" samples, featuring dense packings of individual crystals demonstrate that all C-star samples exhibiting polyhedral aggregates adopt a body centredcubic (BCC) unit cell (Fig. 2b, centre and right). Consistent with a crystal geometry independent on arm length, the measured lattice parameter $a$ increases linearly with $l$, matching very closely the predicted value $a_{\mathrm{t}}$ (Fig. $2 \mathbf{c}$ ). We estimate $a_{\mathrm{t}}$ through a simple geometrical model, where C-star arms are modelled as rigid rods of length equal to $d_{\mathrm{bp}} l\left(d_{\mathrm{bp}}=3.4 \AA / \mathrm{bp}\right)$ and the cholesterol-rich cores are approximated to spheres of radius $r_{\text {mici }}=30.3 \AA$, in very good agreement with the previously estimated value for $l=28$ C-stars ${ }^{32}$

$$
a_{\mathrm{t}}=\frac{4}{\sqrt{5}}\left(d_{\mathrm{bp}} l+r_{\text {mici }}\right) .
$$

The prefactor in Eq. 1 is derived from the hypothesized arrangement of C-stars in the unit cell, where each BCC site is occupied by a hydrophobic core featuring 12 cholesterol molecules, linked by C-Stars as shown in Fig. $2 \mathbf{d}^{32}$ and abstracted in Fig. 2e. A more detailed discussion of the derivation of Eq. 1 can be found in the SI (see Fig. S2). Despite being individually unstable, C-stars with $l=21 \mathrm{bp}$ form a stable crystalline phase, demonstrating how collective interactions further stabilize the DNA motifs. The observed range of viable arm lengths translates into lattice parameters spanning from $18.4 \mathrm{~nm}$ to $30.6 \mathrm{~nm}$, reaching a maximum size well above what is achievable 

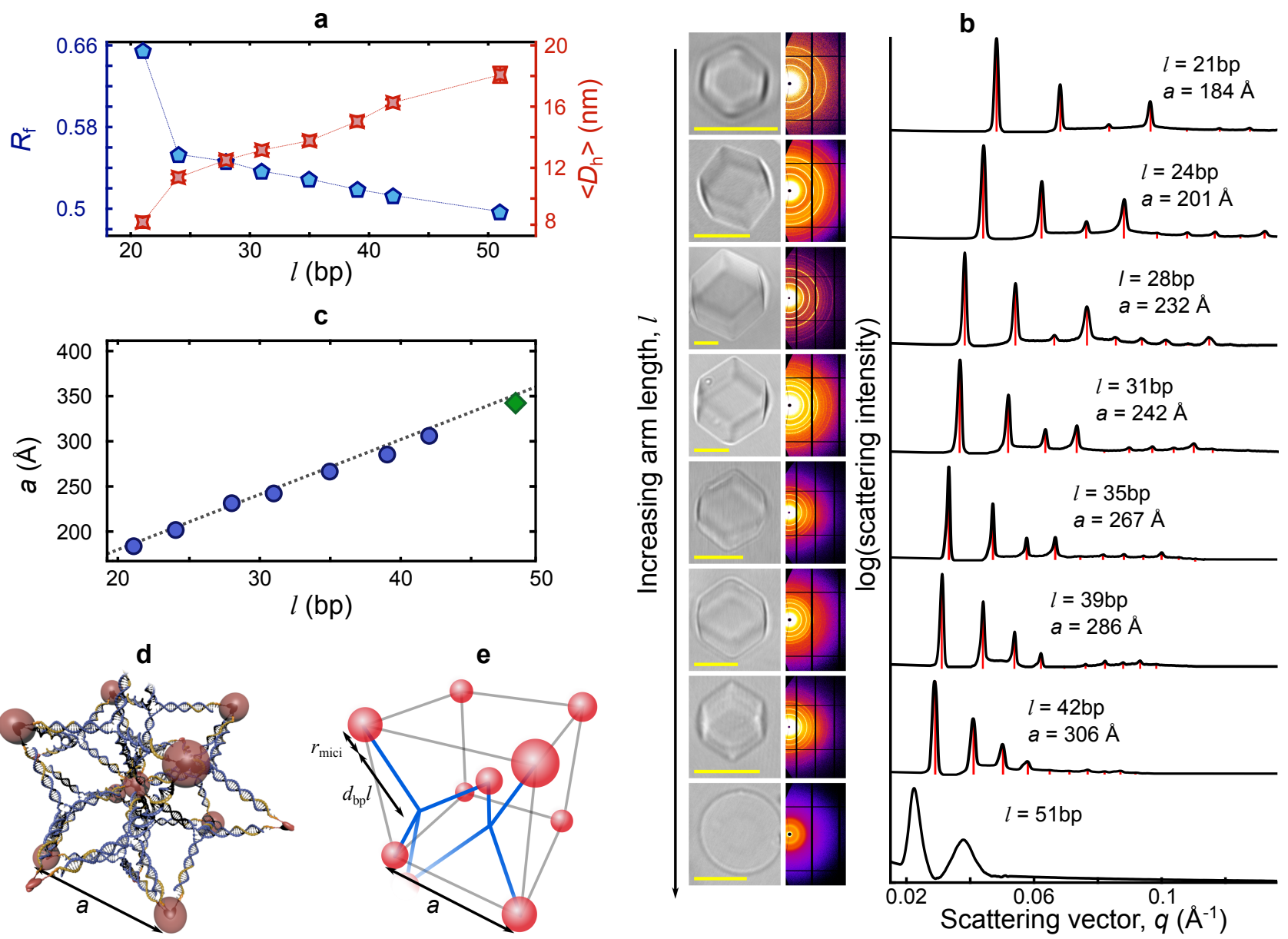

Figure 2: Controlling lattice parameter in C-star crystals. a, Structural characterization of individual nanostars lacking cholesterol functionalization. Increasing $l$ leads to lower migration distance $R_{\mathrm{f}}$ and larger mean hydrodynamic diameter $\left\langle D_{\mathrm{h}}\right\rangle$. b, (left) Bright field images show rhombic-dodecahedral single crystals for $l=21-42 \mathrm{bp}$, and spherical aggregates for $l=51 \mathrm{bp}$. Scale bars are $10 \mu \mathrm{m}$. SAXS powder diffraction patterns reveal that C-stars with arm length $l=21-42 \mathrm{bp}$ form BCC crystalline phases. (Centre) 2D diffraction patterns. (Right) Radially-averaged profiles. Red lines mark best fit to Bragg peaks of a BCC lattice. C-stars with $l=51 \mathrm{bp}$ (bottom) form an amorphous phase. c, Measured lattice parameter increases with $l$, and closely follows the expected value from geometrical arguments (dashed line). Blue circles indicate conventional C-stars, the green diamond the responsive design (Fig. 6). d, Plausible arrangement of C-stars in a BCC unit cell. ${ }^{32} \mathbf{e}$, Abstracted view of unit cell. Here, solid blue lines represent DNA duplexes and red spheres represent micelles. For ease of visualization, only 2 nanostars are shown.

with simple all DNA motifs. ${ }^{20,28}$

Relative peak heights in Fig. 2b gradually change as arm length is increased, implying a change in the electron density distribution within the BCC unit cell. This is to be expected as an increase in $l$ results in a greater fraction of the total material being located within the nanostar arms rather than elsewhere.

The diffraction resolution of C-star frameworks reaches $4 \mathrm{~nm}$, and is limited by the intrinsic flexibility of the nanostar motifs and the non- specificity of hydrophobic interactions that, along with the free swivelling motion of the cholesterol moieties, relax any constraints on the rotational orientation of individual C-stars. Despite limiting diffraction resolution, this inherent flexibility and rotational degeneracy is the primary cause underlying the insensitivity of C-star self-assembly to fine features, which ultimately enables the design versatility discussed in this paper.

Samples with $l=51$ bp display no crystallinity, 
forming instead an amorphous phase (Fig. 2b). We ascribe this behavior to the increased flexibility of the longer DNA arms, which may be too pliant to sustain a rigid network. ${ }^{34}$
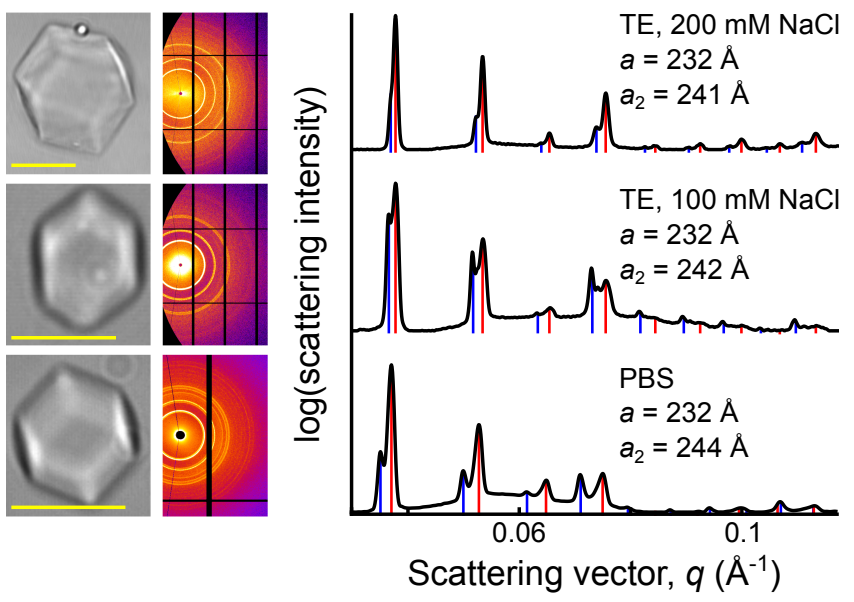

Figure 3: Self-assembly at low ionic strength. (left) Bright field images show rhombic-dodecahedral single crystals formed by $l=28 \mathrm{bp}$ C-stars in low ionic strength buffers. (centre) 2D diffraction patterns. (right) Radially-averaged profiles. Red lines mark the best fit to the Bragg peaks of a BCC lattice with identical lattice parameter as for the frameworks formed by $l=28 \mathrm{bp}$ C-stars in $300 \mathrm{mM} \mathrm{NaCl}$ (2b). Blue lines mark best fit to Bragg peaks of a secondary BCC phase with lattice parameter $a_{2}$. Scale bars $10 \mu \mathrm{m}$.

\section{Self-assembly at low ionic strength.}

Due to the low packing density of C-star frameworks, self-assembly does not require the high cation concentration or divalent ions needed for compact DNA crystals and origami. ${ }^{20,28,31}$ When not stated otherwise, all experiments were performed with a sodium concentration of $300 \mathrm{mM}$ and no divalent ions. In Fig. 3 we show that crystallization is robust even at lower monovalent salt concentration, with well defined single crystals observed in $200 \mathrm{mM}$, $100 \mathrm{mM} \mathrm{NaCl}$, and in physiological phosphate buffered saline (PBS). In all cases, SAXS demonstrates unchanged crystal symmetry and scattering resolution compared with samples prepared in $300 \mathrm{mM} \mathrm{NaCl}$. Besides the BCC phase with lattice parameter identical to the one found in higher ionic-strength conditions, when reducing sodium concentration we observe the emergence of a secondary BCC phase with slightly expanded lattice parameter. We hypothesize that the secondary phase may appear due to conformational changes of the nanostar junction following reduced electrostatic screening. ${ }^{35,37-41}$

The robustness of C-star self-assembly also relaxes the strict need for using highly purified DNA oligonucleotides, which is standard in most DNA nanotechnology preparations. All but the cholesterol functionalized oligonucleotides in our experiments are purified (by the manufacturer) via conventional desalting, significantly reducing costs compared to purification steps based on High-Performance Liquid Chromatography or Polyacrylamide Gel Electrophoresis.

\section{Tuning molecular partitioning}

The conservation of crystal geometry for Cstars with widely different arm length offers a powerful route for the preparation of nanoporous materials with programmable porosity, which we characterize by determining the degree of penetration of various (macro)molecular probes within the crystals.

We chose a variety of fluorescent probes to cover a broad range of molecular weight, hydrodynamic size, hydrophobicity, and chemical nature, including sodium fluorescein (FAM), fluorescein-modified dextrans (3kDa DXTFAM, 10kDa DXT-FAM), rhodamine B, recombinant green fluorescent protein (rGFP), and fluorescently labelled streptavidin (SAvA647). Crystals were soaked in solutions containing the (macro)molecules, and their penetration was determined by extracting the ratio $\xi=I_{\text {internal }} / I_{\text {external }}$ between the fluorescence intensity measured inside and outside the crystals from confocal micrographs.

Macromolecule partitioning is clearly dependant on $l$, as shown by Fig. $4 \mathbf{a}, \mathbf{b}$, with all of the hydrophilic probes trialled, namely FAM, DXT FAM and rGFP, following the trend of longer arm length leading to increased penetration. 

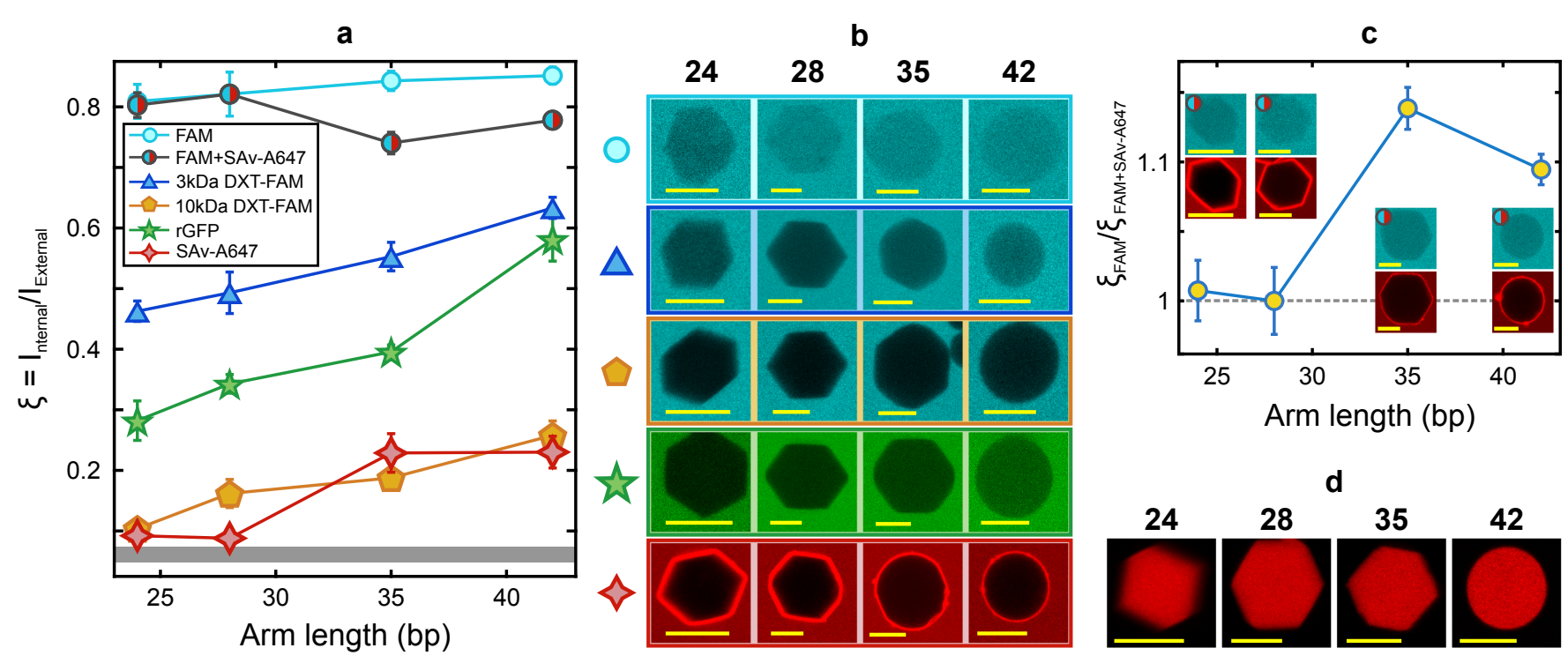

Figure 4: Controllable porosity and macromolecular partitioning in C-star networks. The following fluorescent probes were tested: sodium fluorescein (FAM), fluorescein-labelled 3kDa dextran (3kDa DXT-FAM), fluorescein-labelled 10kDa dextran (10kDa DXT-FAM), recombinant GFP (rGFP), Alexa647-labelled streptavidin (SAv-A647). a, Probe penetration was assessed by confocal microscopy, by measuring the ratio $\xi$ between the mean fluorescence levels inside and outside individual crystals soaked in solutions containing the probes. The grey band marks the level of background signal. b, Confocal micrographs of representative samples. c, Ratio between the $\xi$-values measured for FAM in pristine crystals and crystals previously incubated with SAvA647. d, Confocal micrographs of crystals soaked in rhodamine B. All scale bars are $10 \mu \mathrm{m}$.

As expected, larger molecular weight leads to lower penetration for a fixed $l$. The penetration coefficient of fluorescein can be used to estimate the accessible free volume of the framework as discussed in Ref. ${ }^{32}$ For $l=42 \mathrm{bp}$ the fraction of free volume reaches $85 \%$, a value well above what can be reached with alternative nanoscale DNA building blocks ${ }^{28}$ and that compares favourably even against ultrahigh porosity metal-organic frameworks. ${ }^{42}$

Large SAv molecules are completely excluded from frameworks with $l=24$ and $28 \mathrm{bp}$, for which $\xi$ values are not significantly higher than the measured background, indicating no loading into crystals. A sharp penetration transition is then observed upon increasing $l$ to 35 and $42 \mathrm{bp}$, which produce similar values of $\xi$. This indicates that the pores in the $l=24$ and $28 \mathrm{bp}$ crystals are too small to accommodate the rather bulky protein, whereas the larger pores of the $l=35$ and $42 \mathrm{bp}$ crystals can. As an additional check to show that SAv was completely excluded from crystals with smaller pore size, we added FAM to each of the samples previously exposed to SAv. As shown in Fig. 4a (bi-color symbols), and further highlighted in Fig. 4c, C-stars with $l=35$ and $42 \mathrm{bp}$ show a decrease in the $\xi$ measured for FAM in crystals with SAv in comparison to crystals not soaked in streptavidin - a clear proof that the bulky protein is excluding a fraction of the available free volume to fluorescein. For samples with $l=24$ and $28 \mathrm{bp}$, no change in $\xi$ is observed, confirming that streptavidin does not penetrate these frameworks. Note that SAv displays an affinity for the surface of the crystals and coats them even in conditions in which the mesh size is too small to allow penetration (Fig. 4b). The affinity is likely due to hydrophobic patches on the proteins.

Rhodamine B, a small hydrophobic molecule, was observed to strongly partition within the crystals regardless of arm length, likely due to an affinity for the cholesterol-rich cores, as is clearly visible in Fig. 4d. 


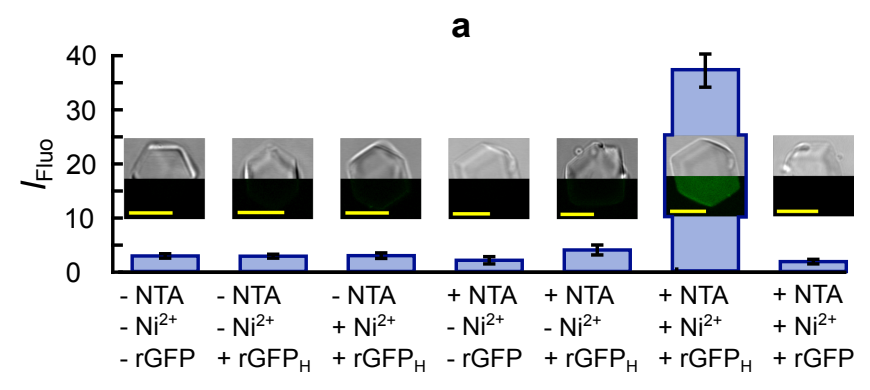

b
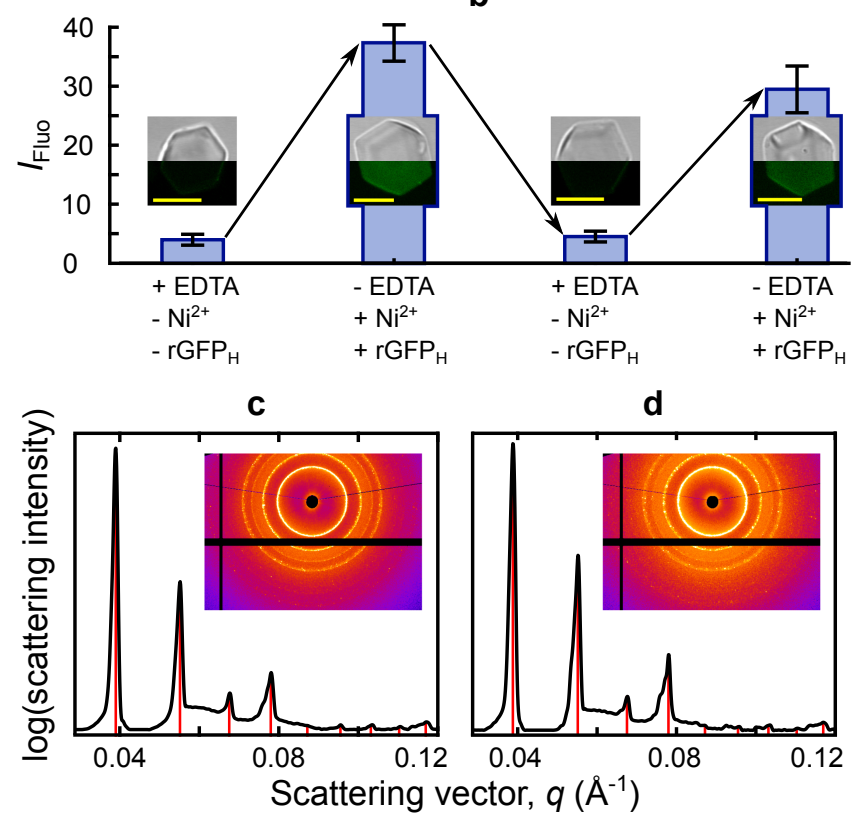

Figure 5: Specific and reversible binding of a target protein. a, Binding of N-terminal $6 \times$ histidine tagged $\mathrm{rGFP}\left(\mathrm{rGFP}_{\mathrm{H}}\right)$ and $\mathrm{rGFP}$ lacking a histidine tag (rGFP) to inert and nitrilotriacetic acid (NTA) functionalized crystals was assessed by measuring the mean fluorescence intensity within crystals, $I_{\text {Fluo }}$, from confocal micrographs. Insets show combined bright field (top half) and fluorescent (bottom half) micrographs of representative crystals. Scale bars $10 \mu \mathrm{m}$. b, Reversibility of $\mathrm{rGFP}_{\mathrm{H}}$ binding to NTA functionalized crystals upon addition and removal of a competing chelating agent demonstrated through confocal microscopy. Insets and scale bars as for a. c, SAXS diffraction patterns of NTA functionalized C-star frameworks. Modified networks adopt the same BCC lattice as inert C-stars. d, After treating with $\mathrm{rGFP}_{\mathrm{H}}$ the structure of NTA C-star aggregates remains the same. For $\mathbf{c}$ and $\mathbf{d}$, red lines indicate the best fit to Bragg peaks of a $\mathrm{BCC}$ lattice.

\section{Specific and reversible entrapment of a target protein}

Although non-specific entrapment of functional proteins has been successfully demonstrated in all-DNA 3D crystals, ${ }^{43}$ site specific positioning has been achieved only in 2D DNA architectures. ${ }^{44-46}$ Owing to their controllable porosity and resilience to changes in building-block design, C-star frameworks offer an attractive platform for site-selective entrapment of functional macromolecules, including proteins, in 3D. Following the strategy applied for 2D nanostructures, ${ }^{44-46}$ to obtain protein-binding capability in 3D frameworks we designed a C-star variant with $l=28$, in which one arm was modified to host a nitrilotriacetic acid (NTA) molecule (see Fig. 1c(ii)). When charged with $\mathrm{Ni}^{2+}$ ions, NTA can specifically bind recombinant proteins featuring poly-histidine residues (His-tags).

As shown in Fig. 5a, NTA-functionalized Cstars form macroscopic single crystals visually similar to their non-functional counterparts. Confocal microscopy demonstrates that Nterminal poly-histidine tagged $\mathrm{rGFP}\left(\mathrm{rGFP}_{\mathrm{H}}\right)$ strongly partitions within crystals previously washed with a $\mathrm{NiCl}_{2}$ solution. Control experiments show that samples lacking either NTA functionalization, $\mathrm{NiCl}_{2}$ or protein exhibit no fluorescence signal under identical imaging conditions. Additionally, no fluorescence signal was detected when performing the experiment using a protein lacking the His-tag (rGFP), fully demonstrating the specificity of the partitioning. Fig. 5b shows that protein entrapment can be controllably reversed by incubating the crystals loaded with $\mathrm{rGFP}_{\mathrm{H}}$ with the chelating agent ethylenediaminetetraacetic acid (EDTA), which strips the nickel from the NTA. The protein can again be captured upon removal of EDTA and addition of new $\mathrm{NiCl}_{2}$. Diffraction patterns obtained from non-treated NTA C-star frameworks (Fig. 5c) and from those treated with $\mathrm{Ni}$ and loaded with $\mathrm{rGFP}_{\mathrm{H}}$ (Fig. 5d) show the same BCC symmetry and identical lattice parameter as the inert C-star design with the same arm length, demonstrating that neither including the NTA moiety nor trapping the protein affect the microstructure of the frame- 
works.

\section{Isothermal dissociation of C-star crystals by strand displacement}

To further demonstrate the robustness of Cstar self-assembly to topology-preserving design changes, we produced a C-star variant featuring a responsive motif. Relying on toeholdmediated strand displacement, ${ }^{47}$ we can induce the isothermal melting of these modified C-star frameworks upon exposure to a trigger oligonucleotide. ${ }^{48,49}$ The potential of strand displacement reactions as a means of controlling structural rearrangements in materials has been demonstrated in arrays of DNA-functionalized gold nanoparticles, ${ }^{50} \mathrm{col}-$ loids, ${ }^{48}$ and liposomes. ${ }^{51}$ However, previous attempts to program a triggered response in all-DNA crystals have been limited to the grafting/displacement of fluorescent DNA oligos, producing color changes but no structural response. ${ }^{52}$

Figure $6 \mathbf{a}$ shows a schematic of the implemented strand displacement mechanism, leading to dissolution of the C-star network. This C-star variant is designed such that the core strands (blue in Fig. 6a) are not bound directly to the cholesterol-functionalized strands (orange in Fig. 6a). Instead, a bridge strand (green in Fig. 6a) connects the nanostar core to the cholesterolized strands, while presenting a dangling 6 nucleotide toe-hold. Upon the addition of the trigger strand (red in Fig. 6a), which is fully complementary to the bridge strand, the core and cholesterolized strands are displaced leaving cholesterol-DNA micelles, free nanostars, and a double stranded waste product composed of the fully hybridized trigger and bridge strands (bottom-left panel, Fig. 6a). The correct functionality of the triggered disassembly mechanism was demonstrated in freely diffusing nanostars using DLS and a fluorescence quenching assay (see SI text and Fig. S3).

The incorporation of the strand displacement motif leads to two key changes in the structure of C-stars, compared to the conventional design. Namely, two nicks are present in the arm-forming duplex rather than one, and there is a 6-base ssDNA dangle one-quarter of the way along the arm. Remarkably, even with these substantial changes, the system retains the same BCC crystalline symmetry of conventional C-stars, as demonstrated by SAXS (black trace, Fig. 6c). The measured lattice parameter, reaching $a=34.2 \mathrm{~nm}$, further extends the range demonstrated with inert C-stars, while being equally well predicted by Eq. 1 (green diamond in Fig. 2c), confirming that the crystal structure is fully conserved.

Trigger-induced isothermal melting of the frameworks is demonstrated by direct visualization of the aggregates, which rapidly dissolve upon exposure to the trigger (time series in Fig. 6b). The same process, is tracked by SAXS, where we observe progressive loss of crystalline order, demonstrated by the broadening first, and then disappearance of the BCC Bragg peaks (Fig. 6c).

\section{Conclusions}

In summary, we introduced a novel approach for the preparation of 3D crystalline frameworks with pre-designed structural features and embedded responsiveness, programmable through straightforward design variations. The flexibility of our approach derives from the selfassembly mechanism of amphiphilic C-stars, in which network geometry depends on the general topology and symmetry of DNA motifs, rather than on high-resolution molecular details. This feature enables unprecedented design freedom, that we exploit to produce a set of geometrically identical frameworks with finely controlled lattice parameters spanning a range of nearly $16 \mathrm{~nm}$ and reaching a maximum of $34.2 \mathrm{~nm}$. By exploiting the resulting high porosity, the controllable mesh size, and the inherent amphiphilic properties of our material, we are able to fine-tune the partitioning of macromolecules within the frameworks based on size and hydrophobicity. Furthermore, the resilience of the self-assembly mechanism, and the significant free volume available within Cstar networks, allowed us to include a respon- 

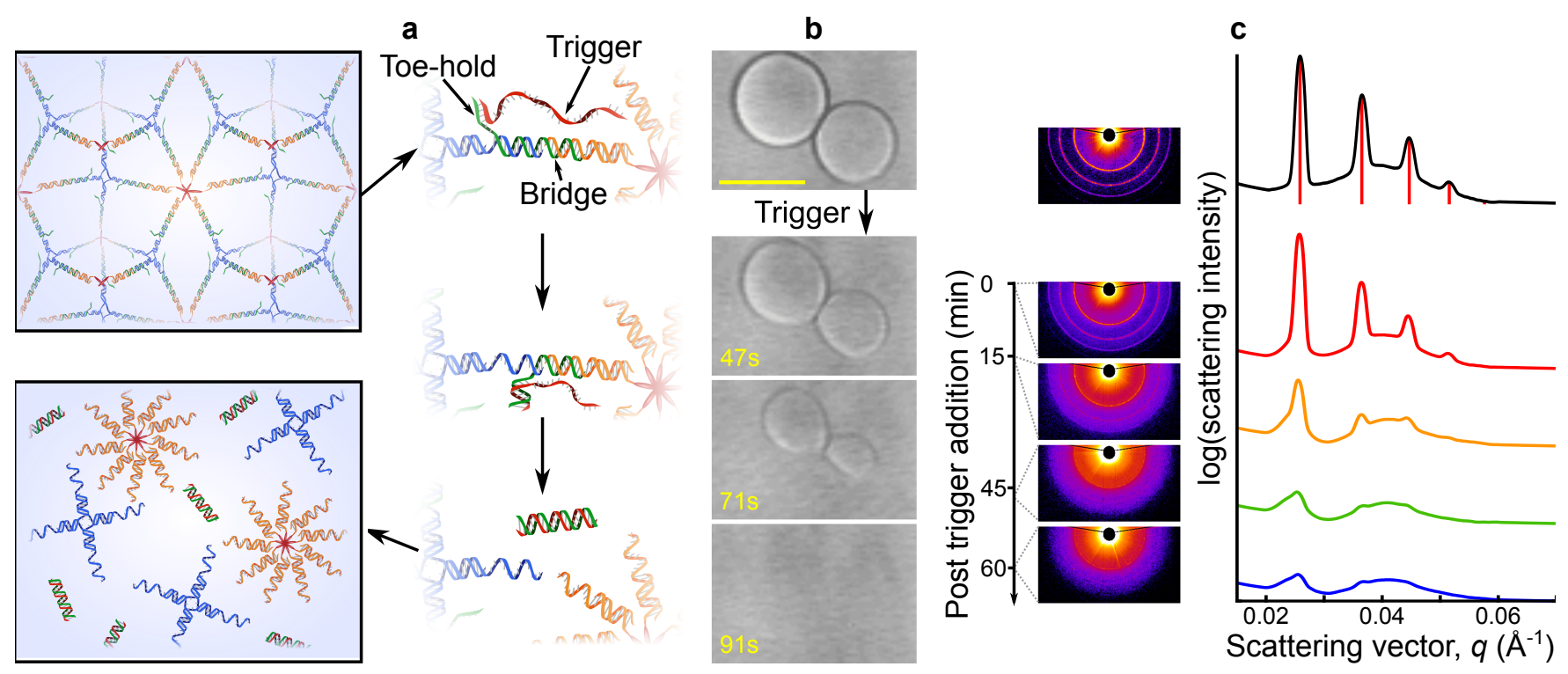

Figure 6: Embedded responsiveness enables isothermal melting of C-star crystals. a, Schematic of the mechanism underlying isothermal melting of C-star crystals. Addition of a trigger strand (red) causes the chol-DNA micelles to dissociate from the nanostar core through toe-hold mediated strand displacement. $\mathbf{b}$, Series of bright-field images showing rapid melting of C-star aggregates upon exposure to the trigger strand. Scale bar $10 \mu \mathrm{m}$. c, Despite the spherical macroscopic appearance, SAXS patterns reveal that aggregates formed by responsive C-Stars are indeed crystalline (top). The radially-averaged diffraction profile (black solid line) is compatible with a BCC symmetry. Red lines indicate the best fit to the Bragg peaks of a BCC lattice. Adding the trigger strand to the SAXS sample induces progressive disruption of crystalline order (coloured solid lines). The slower melting rate compared to that measured in microscopy experiments (b) is a consequence of the slow passive diffusion of the trigger strand through the large sample used for SAXS (see Methods section).

sive DNA motif leading to isothermal dissolution of the frameworks, as well as a chemical modification enabling specific entrapment and triggered release of functional proteins. The structural responsiveness and programmability of the frameworks, combined with their specific and non-specific affinity for various cargoes, hints at the possible application of the material to smart nanomedical vectors. Compared to standard formulations based on lipids, ${ }^{53,54}$ carriers prepared from C-stars could be more easily functionalized with ligands or DNA aptamers to achieve selective targeting of diseased cells, ${ }^{53}$ and programmed to dissolve and release their cargo only in the presence of specific molecular cues, e.g. disease-related microRNAs. ${ }^{55}$ Critical for this and other biomedical applications is also the resilience of C-star frameworks to low and physiological ionic strength. More complex C-Star variants could also carry nucleic acid complexes designed to amplify signals, ${ }^{56,57}$ perform computation, ${ }^{58,59}$ and respond to molecular and environmental cues, ${ }^{60-65}$ opening a new route for producing highly concentrated, well-ordered, sensing/signal-processing architectures with potential applications in point-ofcare diagnostics, nanomedicine, or bottom-up synthetic biology. Finally, C-star self-assembly occurs in a one-pot reaction, and does not require the use of highly purified oligonucleotides, substantially reducing costs and simplifying preparation protocols compared to other available DNA-tile architectures.

Acknowledgement LDM, PC and NJB acknowledge support from the EPSRC Programme Grant CAPITALS number $\mathrm{EP} / \mathrm{J} 017566 / 1$. LDM acknowledges support from the Leverhulme Trust and the Isaac Newton Trust through an Early Career Fellow- 
ship (ECF-2015-494) and from the Royal Society through a University Research Fellowship (UF160152). RAB acknowledges support from the EPSRC CDT in Nanoscience and Nanotechnology (NanoDTC), grant number EP/L015978/1. We acknowledge Diamond Light Source for provision of synchrotron beamtime (SM17271 and SM19371) and we would like to thank A. Smith and T. Snow for assistance in operating beamline I22. We thank T. Knowles and B. Mognetti for insightful discussions on this project.

Supporting Information Available: The following files are available free of charge.

Supplementary methods, figures and DNA sequences.

This material is available free of charge via the Internet at http://pubs.acs.org/.

\section{Methods}

\section{C-Star design and oligonulceotide preparation}

C-Star structures were designed using NUPACK. ${ }^{66}$ Sequences are shown in Tables S1 and S2 of the SI. The nitrilotriacetic acid (NTA) functionalized strand was purchased from Eurogentec, and purified by the supplier using high-performance liquid chromatography (HPLC). All other oligonucleotides were purchased from Integrated DNA Technologies (IDT). Non-functionalized strands were purified by the supplier using standard desalting, while cholesterol-functionalized strands were purified by HPLC. Cy3 and Black Hole Quencher $囚-2$ functionalized strands used for characterizing responsive C-stars (see SI) were purified by HPLC. As received dehydrated DNA was reconstituted in TE buffer $(10 \mathrm{mM}$ Tris, $1 \mathrm{mM}$ EDTA, pH 8.0, Sigma Aldrich). All buffers were syringe-filtered through $0.22 \mu \mathrm{m}$ pore size polyethersulfone filters (Millex) prior to use. The concentration of reconstituted DNA strands was determined by measuring absorbance at $260 \mathrm{~nm}$ using a ThermoScientific Nanodrop 2000 UV-Vis spectrophotometer. Extinction coefficients for all strands were provided by the supplier.

\section{Free nanostar characterization}

Folding of nanostars was assessed using agarose gel electrophoresis (AGE) and dynamic light scattering (DLS). For these experiments the cholesterol-modified strand was replaced with a non-functionalized oligonucleotide of identical sequence to prevent aggregation.

Mixtures of required oligonucleotides were prepared in Eppendorf tubes to give a final nanostar concentration of $10 \mu \mathrm{M}$. Prepared mixtures were cooled from $95^{\circ} \mathrm{C}$ to $20^{\circ} \mathrm{C}$ at $-0.05^{\circ} \mathrm{C} \mathrm{min}^{-1}$ using a Techne ${ }^{\mathrm{TM}}$ TC-512 thermocycler to enable nanostructure hybridization. Annealed samples were stored at $4^{\circ} \mathrm{C}$ and used for electrophoresis and light scattering studies within a week.

Agarose gels were prepared at $1.5 \mathrm{wt} \%$ agarose in Tris-Borate-EDTA buffer (Sigma Aldrich), and precast with DNA stain (SYBR Safe, ThermoFisher). Annealed nanostars were mixed with loading dye (Thermo Scientific) and a volume equivalent to $7.5 \mu \mathrm{g}$ of DNA was loaded into each well. A potential of $75 \mathrm{~V}\left(3 \mathrm{~V} \mathrm{~cm}^{-1}\right)$ was applied for 120 minutes. Gels were imaged using a GelDoc-It imaging system equipped with a UV lamp for illumination and CCD camera for image acquisition.

DLS measurements were performed on a Malvern Zetasizer NanoZSP, equipped with a $633 \mathrm{~nm}$ He-Ne laser with a maximum power of $10 \mathrm{~mW}$. The scattering angle was fixed at $173^{\circ}$. An ultra low volume quartz cuvette (ZEN2112, Malvern) was filled with $50 \mu \mathrm{L}$ of sample syringe-filtered through $0.22 \mu \mathrm{m}$ polyvinylidene fluoride filters (Millex), and sealed with a polypropylene cap to prevent evaporation. Size distributions shown in Fig. 1 are averaged over 3 runs, each of which consisted of 13-16 individual measurements. A Peltier heating block was used for temperature control.

\section{Preparation of C-star crystals}

Samples with C-star concentration of $5 \mu \mathrm{M}$ were prepared by mixing all required strands in 
equimolar quantities in TE buffer with $300 \mathrm{mM}$ $\mathrm{NaCl}$. Samples were loaded into borosilicate glass capillaries with internal section of $4 \times 0.2 \mathrm{~mm}^{2}$ (CM Scientific). Capillaries were cleaned by first sonicating in $2 \%$ Hellmanex III water solution (HellmaAnalytics) at $90^{\circ} \mathrm{C}$ for 15 minutes. Surfactant was removed by two rounds of thorough rinsing and sonication in ultra-pure water (Milli-Q). Prior to filling capillaries, oligonucleotide mixtures prepared in Eppendorf tubes were heated to $95^{\circ} \mathrm{C}$ using a heating block to ensure complete denaturation of any aggregates formed upon initial mixing. After loading into capillaries, a small amount of sample was removed and either side was capped off with mineral oil before sealing permanently onto a microscope slide with epoxy glue (Araldite). Sealed capillaries were then loaded into a fully programmable, Peltier controlled, water bath and heated to $95^{\circ} \mathrm{C}$ for 30 min, before cooling to $20^{\circ} \mathrm{C}$ at $-0.01^{\circ} \mathrm{C} \mathrm{min}{ }^{-1}$.

\section{Imaging and solute-penetration as- says}

Confocal images were obtained using a Leica TCS SP5 microscope equipped with a HC PL APO CORR CS $40 \times / 0.85$ dry objective (Leica). For experiments on macromolecular partitioning, a small volume $(2-5 \mu \mathrm{l})$ of $\mathrm{C}$ stars extracted from capillaries prepared as described above was loaded into a glass microwell slide (Hamamatsu) containing $20 \mu \mathrm{l}$ of fluorescent macromolecule prepared in TE buffer with $300 \mathrm{mM} \mathrm{NaCl}$. Samples were sealed with DNase free tape (Grace Bio-Labs FlexWell SealStrips) to prevent evaporation, and left overnight to equilibrate. For excitation of sodium-fluorescein (FAM, Sigma Aldrich), fluorescein dextran conjugates (3kDa DXT-FAM \& 10kDa DXT-FAM, ThermoFisher), and recombinant green fluorescent protein (rGFP, Sigma Aldrich) an Ar-ion laser (488 nm) was used, for excitation of rhodamine B (RhodB, SigmaAldrich) and Ar-ion laser (514 nm) was used, and a HeNe laser $(633 \mathrm{~nm})$ was used to image Alexa Fluor ${ }^{\mathrm{TM}} 647$ conjugated streptavidin (SAv-A647, ThermoFisher) containing samples. For each sample, we recorded confocal z-stacks of slice thickness $0.5 \mu \mathrm{m}$ of $20-40$ fields of view, each containing between 2-20 crystals. Penetration was quantified by the ratio of mean fluorescence intensity inside to outside the crystals, $\xi$, which was determined through manual masking of objects at their equitorial plane using the BioFormats plugin for ImageJ. ${ }^{67}$ The background level for $\xi$ (grey band in Fig. 4a) was determined using solute-impermeable silica beads as reference (diameter $20 \mu \mathrm{m}$ ).

For experiments on trigger-induced isothermal melting (Fig. 6), samples were initially prepared as for all other C-star variants. A volume of trigger strand equivalent to a 1:1 molar ratio of trigger:bridge strand was added carefully in order not to disturb sedimented crystals. Directly after addition, a time series was recorded at 0.8 frames per second in transmission bright-field mode.

\section{Protein entrapment assay}

Entrapment of N-terminal $6 \times$ His tagged rGFP $\left(\mathrm{rGFP}_{\mathrm{H}}\right.$, ThermoFisher), and rGFP lacking a His-tag (rGFP, Sigma Aldrich), within NTA functionalized C-star networks was characterized using a confocal microscopy assay with the same confocal setup as for solute penetration assays. An Ar-ion laser (488 nm) was used for excitation. Crystals prepared and extracted as described previously were first washed in order to remove EDTA present in the incubation buffer. This was achieved by 5 rounds of centrifugation, supernatant removal, and addition and mixing with washing buffer, WB $(10 \mathrm{mM}$ tris, $300 \mathrm{mM} \mathrm{NaCl}$, pH 8.0). For imaging, a small volume (approx. $5 \mu \mathrm{L}$ ) of washed crystals was added to a glass microwell slide. $\mathrm{Ni}^{2+}$ ions were introduced by addition of $10 \mu \mathrm{L} \mathrm{NiCl}_{2}$ containing WB $\left(10 \mathrm{mM} \mathrm{NiCl}_{2}, \mathrm{WB}\right)$. After allowing to incubate for 1 hour, excess $\mathrm{Ni}^{2+}$ was removed by 5 rounds of washing. Each washing step involved the removal of $10 \mu \mathrm{L}$ of solution from the well, addition of $10 \mu \mathrm{L}$ of fresh WB, and thorough mixing by pipette aspiration. A 5 minute rest between washing steps was included to allow any dispersed crystals to sediment. After the final wash step, $5 \mu \mathrm{L}$ of either $\mathrm{rGFP}_{\mathrm{H}}$ incubation buffer $\left(7 \mu \mathrm{MrGFP} \mathrm{H}_{\mathrm{H}}, 1 \mathrm{mg} \mathrm{ml}{ }^{-1}\right.$ bovine 
serum albumin (BSA), WB), or rGFP incubation buffer $\left(7 \mu \mathrm{M} \mathrm{rGFP,} 1 \mathrm{mg} \mathrm{ml}^{-1}\right.$ bovine serum albumin (BSA), WB) was added. Following a 1 hour incubation, excess protein was removed by 5 rounds of washing with WB. For the demonstration of the reversibility of protein binding (Fig. 5b), bound $\mathrm{rGFP}_{\mathrm{H}}$ was removed by 5 rounds of cleaning with EDTA containing washing buffer (1 mM EDTA, WB). Following this, crystals could be recharged with rGFP $_{\mathrm{H}}$ by repeating the same washing, nickelation, and $\mathrm{rGFP}_{\mathrm{H}}$ addition steps detailed above. Between washing steps and during incubation periods, sample chambers were sealed with DNase free tape to prevent evaporation. Fluorescent and bright field confocal micrographs taken at the equatorial plane of crystals were obtained throughout the process. The degree of protein binding was estimated by taking the mean fluorescent intensity within the crystals, $I_{\text {Fluo, }}$, through manual masking of objects using the BioFormats plugin for ImageJ. ${ }^{67}$

\section{Small angle X-ray scattering}

SAXS measurements were performed at the I22 beamline of the Diamond Light Source using a radiation wavelength of $\lambda=1 \AA$, and beam dimensions of approximately $300 \mu \mathrm{m}$ wide $\times$ $100 \mu \mathrm{m}$ high. The accessible $q$ range was between $5 \times 10^{-3}$ and $0.56 \AA^{-1}$. Here the scattering $q$ vector is defined as $q=(4 \pi / \lambda) \sin \theta \equiv$ $2 \pi / d$, where $d$ is the $d$-spacing, $2 \theta$ is the scattering angle. The $q$-scale was calibrated using silver behenate, which has a well defined layer spacing of $58.38 \AA$.

C-star crystals were prepared in capillaries as described above. The content of 6 capillaries per sample was extracted and concentrated by centrifugation and supernatant removal to a final C-star concentration of $\sim 100 \mu \mathrm{M}$. Concentrated C-Star samples were injected into borosilicate glass X-ray capillaries (diameter 1.6 $\mathrm{mm}$ ) and left to sediment until a clear pellet became visible. Measurements were taken by scanning the X-ray beam across the sample near the pellet region, taking 1 frame per location with an exposure time of $100 \mathrm{~ms}$. Averaged diffraction patterns were built up from at least
20 locations per sample. Background subtraction was performed in two steps for the SAXS patterns shown in Fig. 2, Fig. 5 and Fig. 6 . Measured scattering from the buffer was first subtracted, followed by subtraction of an arbitrary logarithmic background to account for variable scattering between glass capillaries.

For experiments on trigger-induced isothermal melting (Fig. 6), after an initial measurement, a volume of trigger strand approximately corresponding to a 1:1 molar ratio of trigger:bridge strand was carefully added at the top of the capillary and allowed to passively diffuse throughout the sample. Measurements were taken as for previous samples, with identical scan locations for all time points. Between measurements, care was taken not to move the capillary in order to allow for direct comparison of the same pellet region over time.

For experiments of protein binding in NTA functionalized networks, the same washing and $\mathrm{rGFP}_{\mathrm{H}}$ addition steps as described for the confocal binding assay were used. Here, fresh buffer addition and washing was performed with gel-loading pipette tips (Corning@) to allow for thorough mixing and more efficient buffer extraction from narrow x-ray capillaries.

\section{References}

(1) Seeman, N. C. J. Theor. Biol. 1982, 99, 237-247.

(2) Seeman, N. C. J. Biomol. Struct. Dyn. 1985, 3, 11-34.

(3) Li, H.; Park, S. H.; Reif, J. H.; LaBean, T. H.; Yan, H. J. Am. Chem. Soc 2004, 126, 418-419.

(4) Hariri, A. A.; Hamblin, G. D.; Gidi, Y.; Sleiman, H. F.; Cosa, G. Nat. Chem. 2015, 7, 295.

(5) Rahbani, J. F.; Hariri, A. A.; Cosa, G.; Sleiman, H. F. ACS Nano 2015, 9, 1189811908.

(6) Jones, M. R.; Seeman, N. C.; Mirkin, C. A. Science 2015, $34 \%$. 
(7) Winfree, E.; Liu, F.; Wenzler, L. A.; Seeman, N. C. Nature 1998, 394, 539-544.

(8) Park, S. H.; Pistol, C.; Ahn, S. J.; Reif, J. H.; Lebeck, A. R.; Dwyer, C.; LaBean, T. H. Angew. Chem. Int. Ed. 2006, 45, 735-739.

(9) Hansen, M. N.; Zhang, A. M.; Rangnekar, A.; Bompiani, K. M.; Carter, J. D.; Gothelf, K. V.; LaBean, T. H. J. Am. Chem. Soc 2010, 132, 14481-14486.

(10) Majumder, U.; Rangnekar, A.; Gothelf, K. V.; Reif, J. H.; LaBean, T. H. J. Am. Chem. Soc 2011, 133, 3843-3845.

(11) Selmi, D. N.; Adamson, R. J.; Attrill, H.; Goddard, A. D.; Gilbert, R. J. C.; Watts, A.; Turberfield, A. J. Nano Lett. 2011, 11, 657-660.

(12) Aslan, H.; Krissanaprasit, A.; Besenbacher, F.; Gothelf, K. V.; Dong, M. Nanoscale 2016, 8, 15233-15240.

(13) He, Y.; Chen, Y.; Liu, H.; Ribbe, A. E.; Mao, C. J. Am. Chem. Soc 2005, 127, 12202-12203.

(14) Lee, J.; Hamada, S.; Hwang, S. U.; Amin, R.; Son, J.; Dugasani, S. R.; Murata, S.; Park, S. H. Sci. Rep. 2013, 3, 2115 .

(15) Avakyan, N.; Conway, J. W.; Sleiman, H. F. J. Am. Chem. Soc. 2017, 139, 12027-12034.

(16) Seeman, N. C.; Sleiman, H. F. Nat. Rev. Mater. 2017, 3, 17068.

(17) Ke, Y.; Ong, L. L.; Shih, W. M.; Yin, P. Science 2012, 338, 1177-1183.

(18) Hong, F.; Jiang, S.; Wang, T.; Liu, Y.; Yan, H. Angew. Chem. Int. Ed. 2016, 55, $12832-12835$.

(19) Ong, L. L.; Hanikel, N.; Yaghi, O. K.; Grun, C.; Strauss, M. T.; Bron, P.; LaiKee-Him, J.; Schueder, F.; Wang, B.;
Wang, P.; Kishi, J. Y.; Myhrvold, C.; Zhu, A.; Jungmann, R.; Bellot, G.; Ke, Y.; Yin, P. Nature 2017, 552, 72-77.

(20) Zheng, J.; Birktoft, J. J.; Chen, Y.; Wang, T.; Sha, R.; Constantinou, P. E.; Ginell, S. L.; Mao, C.; Seeman, N. C. $N a-$ ture 2009, 461, 74-77.

(21) Aricò, A. S.; Bruce, P.; Scrosati, B.; Tarascon, J.-M.; van Schalkwijk, W. Nat. Mater. 2005, 4, 366-377.

(22) Zhang, Q.; Uchaker, E.; Candelaria, S. L.; Cao, G. Chem. Soc. Rev. 2013, 42, 31273171.

(23) Flory, F.; Escoubas, L.; Berginc, G. J. Nanophotonics 2011, 5 .

(24) Huang, X.-J.; Choi, Y.-K. Sens. Actuator B-Chem. 2007, 122, 659-671.

(25) Xia, L.; Wei, Z.; Wan, M. J. Colloid Interface Sci. 2010, 341, 1-11.

(26) Jimenez-Cadena, G.; Riu, J.; Rius, F. X. Analyst 2007, 132, 1083-1099.

(27) Han, J.; Fu, J.; Schoch, R. B. Lab Chip 2008, 8, 23-33.

(28) Simmons, C. R.; Zhang, F.; MacCulloch, T.; Fahmi, N.; Stephanopoulos, N.; Liu, Y.; Seeman, N. C.; Yan, H. J. Am. Chem. Soc 2017, 139, 11254-11260.

(29) Manuguerra, I.; Grossi, G.; Thomsen, R. P.; Lyngs $\varnothing$, J.; Pedersen, J. S.; Kjems, J.; Andersen, E. S.; Gothelf, K. V. ACS Nano 2017, 11, 9041-9047.

(30) Douglas, S. M.; Dietz, H.; Liedl, T.; Högberg, B.; Graf, F.; Shih, W. M. Nature 2009, 459, 414-418.

(31) Zhang, T.; Hartl, C.; Frank, K.; HeuerJungemann, A.; Fischer, S.; Nickels, P. C.; Nickel, B.; Liedl, T. Adv. Mater. 2018, 30, 1800273 .

(32) Brady, R. A.; Brooks, N. J.; Cicuta, P.; Di Michele, L. Nano Lett. 2017, 17, 32763281 . 
(33) Biffi, S.; Cerbino, R.; Bomboi, F.; Paraboschi, E. M.; Asselta, R.; Sciortino, F.; Bellini, T. Proc. Natl. Acad. Sci. U. S. A. 2013, 110, 15633-15637.

(34) Rovigatti, L.; Smallenburg, F.; Romano, F.; Sciortino, F. ACS Nano 2014, 8, 3567-3574.

(35) Fernandez-Castanon, J.; Bomboi, F.; Rovigatti, L.; Zanatta, M.; Paciaroni, A.; Comez, L.; Porcar, L.; Jafta, C. J.; Fadda, G. C.; Bellini, T.; Sciortino, F. J. Chem. Phys. 2016, 145 .

(36) Auyeung, E.; Li, T. I. N. G.; Senesi, A. J.; Schmucker, A. L.; Pals, B. C.; de la Cruz, M. O.; Mirkin, C. A. Nature 2014, $505,73-77$.

(37) Cooper, J. P.; Hagerman, P. J. Proceedings of the National Academy of Sciences 1989, 86, 7336-7340.

(38) Duckett, D. R.; Murchie, A. I. H.; Lilley, D. M. J. The EMBO journal 1990, 9 2, 583-90.

(39) Lilley, D.; Clegg, R. Annu. Rev. Biophys. Biomol. Struct. 1993, 22, 299-328.

(40) Seeman, N.; Kallenbach, N. Annu. Rev. Biophys. Biomol. Struct. 1994, 23, 53-86.

(41) Nöllmann, M.; Stark, W. M.; Byron, O. Biophys. J. 2004, 86, 3060-3069.

(42) Furukawa, H.; Ko, N.; Go, Y. B.; Aratani, N.; Choi, S. B.; Choi, E.; Yazaydin, A. Ö.; Snurr, R. Q.; O'Keeffe, M.; Kim, J.; Yaghi, O. M. Science 2010, 329, 424-428.

(43) Geng, C.; Paukstelis, P. J. J. Am. Chem. Soc 2014, 136, 7817-7820.

(44) P., G. R.; M., E. C.; Jonathan, M.; M., H. W.; L., M. M.; N., K. A.; J., T. A. ChemBioChem 2009, 10, 1551-1557.

(45) Wenyi, H.; Jie, C.; Qin, Y.; Shoujun, X. Chinese Journal of Chemistry 2010, 28, 1795-1798.
(46) Shen, W.; Zhong, H.; Neff, D.; Norton, M. L. J. Am. Chem. Soc 2009, 131, 6660-6661.

(47) Zhang, D. Y.; Winfree, E. J. Am. Chem. Soc 2009, 131, 17303-17314.

(48) Tison, C. K.; Milam, V. T. Langmuir 2007, 23, 9728-9736.

(49) Rogers, W. B.; Manoharan, V. N. Science 2015, 347, 639.

(50) Kim, Y.; Macfarlane, R. J.; Jones, M. R.; Mirkin, C. A. Science 2016, 351, 579-582.

(51) Parolini, L.; Kotar, J.; Di Michele, L.; Mognetti, B. M. ACS Nano 2016, 10, 2392-2398.

(52) Hao, Y.; Kristiansen, M.; Sha, R.; Birktoft, J. J.; Hernandez, C.; Mao, C.; Seeman, N. C. Nat. Chem. 2017, 9, 824.

(53) Deshpande, P. P.; Biswas, S.; Torchilin, V. P. Nanomedicine (Lond.) 2013, 8 .

(54) Puri, A.; Loomis, K.; Smith, B.; Lee, J.H.; Yavlovich, A.; Heldman, E.; Blumenthal, R. Crit. Rev. Ther. Drug Carrier Syst. 2009, 26, 523-580.

(55) Jansson, M. D.; Lund, A. H. Mol. Oncol. 2012, 6, 590-610.

(56) Dirks, R. M.; Pierce, N. A. Proc. Natl. Acad. Sci. U.S.A. 2004, 101, 1527515278.

(57) Li, B.; Ellington, A. D.; Chen, X. Nucleic Acids Res 2011, 39.

(58) Stojanovic, M. N.; Mitchell, T. E.; Stefanovic, D. J. Am. Chem. Soc 2002, 124, 3555-3561.

(59) Seelig, G.; Soloveichik, D.; Zhang, D. Y.; Winfree, E. Science 2006, 314, 15851588 . 
(60) Goodman, R. P.; Heilemann, M.; Doose, S.; Erben, C. M.; Kapanidis, A. N.; Turberfield, A. J. Nat. Nano. 2008, 3, 93-96.

(61) Zheng, J.; Li, J.; Jiang, Y.; Jin, J.; Wang, K.; Yang, R.; Tan, W. Anal. Chem. 2011, 83, 6586-6592.

(62) Idili, A.; Plaxco, K. W.; Vallée-Bélisle, A.; Ricci, F. ACS Nano 2013, 7, 10863 10869.

(63) Idili, A.; Vallée-Bélisle, A.; Ricci, F. J. Am. Chem. Soc 2014, 136, 5836-5839.

(64) Amodio, A.; Adedeji, A. F.; Castronovo, M.; Franco, E.; Ricci, F. J. Am. Chem. Soc 2016, 138, 12735-12738.

(65) Wu, N.; Willner, I. Nano Lett. 2016, 16, 6650-6655.

(66) Zadeh, J. N.; Steenberg, C. D.; Bois, J. S.; Wolfe, B. R.; Pierce, M. B.; Khan, A. R.; Dirks, R. M.; Pierce, N. A. J. Comput. Chem. 2011, 32, 170-173.

(67) Linkert, M.; Rueden, C. T.; Allan, C; Burel, J-M.; Moore, W.; Patterson, A.; Loranger, B.; Moore, J.; Neves, C.; MacDonald, D.; Tarkowska, A.; Sticco, C; Hill, E.; Rossner, M.; Eliceiri, K. W.; Swedlow, J. R. J. Cell Biol. 2010, 189, 777-782. 


\section{Graphical TOC Entry}

\begin{tabular}{|c|c|}
\hline $\begin{array}{l}\text { Amphiphilic } \\
\text { DNA motif... }\end{array}$ & Robust to design changes \\
\hline & 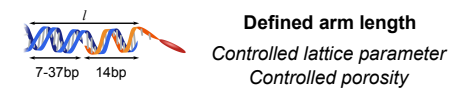 \\
\hline $\begin{array}{l}\text { DNA } \\
\text { single crystals }\end{array}$ & $\begin{array}{c}\text { Chemical modification } \\
\text { Specific \& reversible binding } \\
\text { of target protein }\end{array}$ \\
\hline $30 \mu \mathrm{m}$ & $\begin{array}{c}\text { Embedded functionality } \\
\text { Isothermal melting through } \\
\text { strand displacment }\end{array}$ \\
\hline
\end{tabular}

